\title{
POSIBILIDADES DIDÁCTICAS DE LAS INFRAESTRUCTURAS DE DATOS ESPACIALES PROPUESTA DE APLICACIÓN DIDÁCTICA DE IDERioja
}

\author{
Nuria Pascual Bellido \\ Universidad de La Rioja
}

\begin{abstract}
RESUMEN: El uso de los Sistemas de Información Geográfica (SIG) y otras plataformas para el tratamiento de la información espacial está generando una mejoría en las habilidades cartográficas del alumnado y, en general, en la Competencia digital. Dentro de la amplia oferta existente destacan Google Earth, Géoportail o las Infraestructuras de Datos Espaciales (IDEs). En los últimos años, han ganado terreno estas últimas por las ventajas que ofrecen a la Administración, así como por sus posibilidades didácticas al ayudar al alumno a la comprensión del espacio y, en general, de las relaciones espaciales a todos los niveles y escalas de trabajo. En este artículo se plantea una justificación teórica de la aplicación de la plataforma IDERioja y se presenta una propuesta de actividades en las aulas de Primaria y Secundaria.
\end{abstract}

PALABRAS CLAVE: Sistema de Información Geográfica (SIG), Infraestructura de Datos Espaciales (IDE), didáctica, Educación Primaria, Educación Secundaria Obligatoria.

\section{DIDACTIC POSSIBILITIES OF GEOSPATIAL DATA INFRASTRUCTURES IDERioja IMPLEMENTATION PROPOSAL}

\footnotetext{
ABSTRACT: Geographic Information Systems (GIS) and other platforms for geographic information processing are often used in Geography lessons, as they have led to an improvement in student cartographic skills and, on the whole, in the digital competence. In recent years, Spatial Data Infrastructures (SDIs) have gained ground because of the advantages they offer to state administration as well as their didactic possibilities. In this regard, they bring students up to the comprehension of the space and, in general, of spatial relationships at all levels and work scales. The aim of this paper is to give a theoretical justification of IDERioja platform implementation, as well as, to present a proposal of activities for Primary and Secondary Education.
} 
KEYWORDS: Geographic Information System (GIS), Spatial Data Infrastructure (SID), didactic, Primary school and secondary level.

Recibido: 25/03/2014

Aceptado: 01/09/2014

\section{INTRODUCCIÓN}

Desde hace décadas, la utilización de las Tecnologías de la Información y la Comunicación (TIC) en las aulas ha supuesto una mejoría en el grado de aprendizaje de los alumnos, pero también ha traído consigo el planteamiento de nuevos retos. Son un apoyo incondicional del profesor pero aprender los aspectos técnicos es sólo una pequeña parte del proceso siendo necesario, además, desentrañar sus posibilidades didácticas y pedagógicas. En todas las asignaturas y, por ende en Geografía, el alumno se enfrenta al manejo de Sistemas de Información Geográfica (SIG) y de algunas plataformas para el tratamiento de la información georreferenciada (Capel, 2010), como herramientas de aprendizaje que les acercan a los problemas espaciales (medioambientales y sociales) de una manera más práctica, activa y con una visión más crítica. Su fácil acceso y las posibilidades didácticas que ofrecen, tanto al docente como al discente, han mejorado las habilidades cartográficas del alumno así como su competencia digital (Mejía, 2008; Carceller, Miguel, Losada, Vidal, Gayán y Zaragoza-Soria, 2009; Arranz, Pueyo y Zúñiga, 2011; y De Miguel, De Lázaro y Marrón, 2012). En este sentido, las Infraestructuras de Datos Espaciales (IDEs) están jugando un papel destacado no solo en el ámbito de la Educación sino también en el de la Administración pública. Aunque en este trabajo se va a abordar su aplicación con fines puramente didácticos, se aporta también una breve presentación general del desarrollo de estas plataformas con diferentes fines en España.

\subsection{Las IDEs como instrumento de información geoespacial}

Las IDEs se enmarcan dentro de la Directiva europea INSPIRE (Insfraestructure for Spatial Information in Europe)" cuyo objetivo es "desencadenar la creación de una infraestructura de información espacial que proporcione a los usuarios servicios de información espacial integrados" (Mas, 2002; Mas y Vallejo, 2003; Capdevila i Subirana, 2004) o lo que es lo mismo "hacer que exista una información geográfica armonizada y de alta calidad, asequible y rápidamente disponible para formular, implementar, controlar y evaluar las políticas de la Comunidad Europea, y que permita al ciudadano acceder a la información espacial, ya sea ésta local, regional, nacional o internacional" (Land, 2004; Bernard, Kanellopoulos, Annoni y Smits, 2005). La Directiva INSPIRE es el antecedente directo del proyecto ESDIN (Zapata, 2011)2.

1. Directiva 2007/2/CE del Parlamento Europeo y del Consejo.

2. Este proyecto forma parte de eContentplus, un programa comunitario para producir contenido digital en Europa más accesible y manejable. De este modo, se mejora la calidad del contenido a través de metadatos bien definidos y se refuerza la cooperación entre los agentes implicados. 
Estas plataformas son consideradas por algunos estudiosos como una parte del proceso evolutivo de los SIG. Se componen de datos, metadatos y servicios de carácter local, regional, nacional o global, que ofrecen a la sociedad un conjunto de recursos técnicos para gestionar la información geográfica (Mejía, 2008; Nogueras-Iso, Zaragoza-Soria, Béjar y Muro-Medrano, 2005; Rodríguez, Abad, Alonso, Zamorano, De Carvalho, Caballero y Ceballos-Zúñiga, 2008). Pero, a diferencia de los SIG, las IDEs forman un conjunto de recursos más global, por lo que deberían ser vistas como el núcleo de la geo-informática. Por este motivo y, como se ha citado anteriormente, las administraciones públicas y el ámbito académico son campos en los que su aplicación resulta muy beneficiosa.

Como muestra de su importancia en el ámbito administrativo puede mencionarse el hecho de que la Infraestructura de Datos Espaciales de España (IDEE) ampara las IDEs de las diecisiete comunidades autónomas; a nivel local hay 14 proyectos y a nivel transfronterizo 2 proyectos: OTALEX (Observatorio Territorial y Ambiental para el Alentejo y Extremadura) y SIGNII (IDE para el territorio rural de Galicia y norte de Portugal $)^{3}$.

\subsection{Utilización didáctica de las IDEs}

Las tecnologías de la información geográfica (TIG) han avanzado al mismo ritmo que las TICs y, hoy por hoy, se utilizan de manera habitual para poder actuar de manera más acertada en la solución de los problemas que afectan al medio ambiente y a las sociedades en general. Sin embargo, en opinión de algunos expertos parece que, desde el punto de vista académico, todavía los SIG y las IDEs no se han desarrollado completamente y es necesario que formen parte de la educación en las ciencias y las tecnologías de la información geográfica (Mejía, 2008). No se ha dado este paso a nivel universitario y, mucho menos, en educación primaria y secundaria, niveles en los que los problemas de aprendizaje se multiplican por el grado de desarrollo del alumnado y la dificultad de comprender el espacio abstracto. En países como Estados Unidos se reconocen interesantes innovaciones en algunas escuelas de primaria y secundaria, donde se han adoptado las TIG y los SIG, pero no las IDEs debido a la ausencia de apoyo institucional y de incentivos profesionales, además de las preocupaciones de los profesores por el acceso a la tecnología, la formación y los materiales curriculares. En países latinoamericanos como Colombia, Venezuela, México, Brasil, Argentina o Chile, donde se han definido diferentes niveles de enseñanza de TIG, se reconoce que las IDEs no figuran en los programas académicos porque quienes proponen los planes de estudio y los contenidos de las materias, no están capacitados para manejarlas (Mejía, 2008; González, Gómez, Pérez de los Reyes y Cuzan, 2008). Es decir, el personal docente dedicado a las TIG conoce y aplica bien el lado tecnológico, pero no así, el enfoque pedagógico y didáctico.

En cualquier caso, en las últimas décadas se ha avanzado mucho. De hecho, la bibliografía nacional sobre la aplicación de las TICs en todas las disciplinas en general y en Ciencias Sociales en particular es abundante (Pérez y Vera, 2004; Díez, Gámez,

3. Consultado el 19 de junio de 2014 en, http://www.idee.es/. 
Cruz y Rueda, 2004; Soriano, 2004; Vera y Pérez, 2004; Valvuena y Valverde, 2004; Gómez, 2010; Gómez, 2011, Durán, López, Saavedra, Sánchez y Vilariño, 2011; Velarde et al., 2012; De Alba, García y Santisteban, 2012, entre otros). Además y, por lo que se refiere a las IDEs, el Instituto Geográfico Nacional ofrece una información muy completa e interesante desde el punto de vista didáctico en su página web ${ }^{4}$.

\section{JUSTIFICACIÓN DE LA PROPUESTA DE APLICACIÓN DIDÁCTICA}

\subsection{Objetivos y ámbito de aplicación (antes estaban separados)}

El objetivo de este trabajo es presentar las posibilidades didácticas de las infraestructuras de datos espaciales como instrumento de aprendizaje y ejemplificarlas con la herramienta de geo-visualización IDERioja, mostrando varias actividades prácticas que ayudan a potenciar el tratamiento de la información y la competencia digital en el alumnado.

Los alumnos del siglo XXI presentan unas habilidades increíbles en el manejo de tablets, internet, videojuegos, blogs, webquests y otras herramientas de este tipo y la labor del profesor es encauzar el aprendizaje hacia el objeto de análisis de una manera provechosa y siguiendo unos planteamientos metodológicos, procedimentales y actitudinales adecuados.

Pero en este proceso el papel del docente es fundamental pasando de ser una figura que sólo impone disciplina y genera clases magistrales a ser, además de un modelo, un profesor activo, actor y animador social que interviene de manera puntual en los momentos cruciales del aprendizaje (Delval, 2012; Genovart, 2011; Moraga, 2011).

Son abundantes los trabajos en los que se utilizan los SIGs para explicar a los alumnos determinadas cuestiones que van desde la mera localización espacial, hasta presentar itinerarios didácticos, estudios de paisaje, cuestiones elementales de ordenación del territorio, recorridos urbanos, estudios básicos de impacto ambiental, entre otros ${ }^{5}$. Este tipo de trabajos están pensados especialmente para alumnos de Secundaria, Bachillerato e incluso universitarios. Pero también desde las aulas de Primaria la información que ofrecen las IDEs puede y debe ser utilizada para motivar al alumnado de manera que sepa gestionarla y aprender a interpretarla.

Con la abundante información geográfica que aportan se plantea la posibilidad de diseñar actividades que ayudarán al profesor y al alumno en la tarea de aprender

4. Consultado el 19 de junio de 2014 en, http://www.ign.es/ign/layout/cartografiaEnsenanza.do.

5. Para no hacer un listado exhaustivo que aportaría poco al artículo, mencionamos las publicaciones recogidas en el volumen titulado Aportaciones de la Geografía a lo largo de la vida y editado por la Universidad de Málaga y la AGE, en el Congreso Ibérico de Didáctica de la Geografía; las recogidas en el Cd-rom La educación geográfica digital, del grupo de Didáctica de la AGE y la Universidad de Zaragoza; o las incluidas en otro Cd-rom, Nuevos aires en la Geografía española del siglo XXI, más especializado que los anteriores, y que recoge la contribución española en el $32^{\circ}$ Congreso de Colonia. Finalmente, debe mencionarse el Cd-rom del Congreso Internacional Innovación Metodológica y Docente en Historia, Arte y Geografía, celebrado en Santiago de Compostela en 2011. Todos ellos están debidamente referenciados en la bibliografía final. 
conocimientos relacionados con el entorno natural, social y cultural (identificar los elementos del medio y reconocer las transformaciones a lo largo del tiempo, analizar su organización y características principales, reconocer el impacto antrópico, etc.), así como en la iniciación en las tecnologías de la información, la representación cartográfica y la resolución de problemas que afectan al medio.

La propuesta concreta que se plantea es una aplicación didáctica de IDERioja para el aprendizaje del espacio urbano. En un trabajo anterior presentado por García y Pascual, se planteaba la necesidad de ayudar al alumno a comprender y dar solución a los problemas sociales desde esta disciplina. Se planteaba asimismo la inquietud ante un hecho constatado en las aulas: los alumnos no conocen el espacio en el que se desarrolla su vida cotidiana (García y Pascual, 2012). Lo entienden como un soporte físico sobre el que se instalan las distintas actividades económicas, pero no entienden el espacio como producto social y no saben dar una explicación adecuada al porqué de la distribución espacial de la población y de las actividades, de los desplazamientos cotidianos, de las horas punta de tráfico, del diseño de las edificaciones o del viario. En definitiva, les cuesta entender que la ciudad es un ente vivo y que, como tal, va cambiando de fisonomía con el paso del tiempo y adaptándose con mayor o menor éxito a los "caprichos" de la planificación urbana y del progreso, en general. Sin duda es un tema tan apasionante como complejo y que el alumno suele considerar poco atractivo. Corresponde al docente captar su atención, de manera que sea él mismo el que plantee preguntas, desarrolle su conciencia crítica, busque soluciones y, en definitiva, tire del carro del proceso de enseñanzaaprendizaje. Para el alumno de Secundaria los objetivos son los mismos, aunque haciendo hincapié en procesos urbanos más complejos (evolución de las estructuras urbanas, movilidad, accesibilidad, desequilibrios socioeconómicos, localización de la población inmigrante, etc.).

En definitiva, la propuesta va dirigida concretamente a alumnos de $6^{\circ}$ de Primaria y de $1^{\circ}$ y $3^{\circ}$ de Secundaria. En el diseño de las actividades se ha tenido en cuenta el currículum de cada curso seleccionando los contenidos y las competencias de modo que los resultados del aprendizaje sean acordes con lo exigido en cada nivel. Se trata de analizar el espacio urbano de manera ajustada al nivel formativo del alumnado e intentando mostrar sus características desde diferentes puntos de vista. Para ello se han diseñado una serie de actividades que permitan al alumno mejorar sus habilidades cartográficas y de interpretación del espacio geográfico.

\subsubsection{Contenidos que pueden trabajarse}

En Primaria, se pueden tratar los conjuntos temáticos incluidos en los bloques 1 y 4. En el Bloque 1. Geografía el entorno y su conservación, se estudian la orientación en el espacio, los puntos cardinales y el uso de croquis y mapas, en el Segundo ciclo; y la presentación de espacios conocidos a escala, así como la utilización e interpretación de diferentes representaciones sobre un mismo espacio (planos, fotografías aéreas y otros medios tecnológicos). En el Bloque 4. Personas, culturas y organización social se abordan, en Segundo ciclo, cuestiones sobre las tecnologías de la información y la comunicación, así como la obtención de la información y valoración de contenidos. En Secundaria los contenidos se complican y se distribuyen de otra mane- 
$\mathrm{ra}^{6}$. Por lo que afecta al planteamiento que se está desarrollando y, de modo resumido, pueden citarse los siguientes. En 1o de la ESO entraría el Bloque 1 de contenidos comunes (lectura e interpretación de imágenes y mapas de diferentes escalas; percepción de la realidad geográfica próxima y lejana; interpretación y elaboración de gráficos; obtención de información de fuentes diversas y elaboración escrita de la información obtenida). En 2º de la ESO, el Bloque 1 (búsqueda de información sobre el entorno de fuentes diversas y elaboración escrita de la información obtenida) y el Bloque 2 (la vida en el espacio urbano y aspectos básicos de demografía). En 3o de la ESO, sobre todo, los contenidos comunes del Bloque 1 ; los aspectos relacionados con las actividades económicas (localización y caracterización) en el Bloque 2; y el Bloque 4 en las cuestiones relacionadas con los movimientos migratorios y la desigualdad en el desarrollo humano. En definitiva, en $1^{\circ}$ cuestiones relacionadas con el medio ambiente; en $2^{\mathrm{o}}$ cuestiones de ocupación del espacio y procesos territoriales y en $3^{\circ}$ se podrían retomar aspectos de los cursos anteriores para estudiarlos con más profundidad y, además, los temas de organización territorial y desequilibrios territoriales.

\subsubsection{Competencias que desarrollan}

Antes de abordar las competencias, hay que hacer referencia a un hecho fundamental que tiene que ver con la Geografía y las TICs. En la actualidad existe un debate, más o menos claro, acerca de la necesidad de la revisión curricular de la Geografía. De hecho, parece que debe hacer frente a cuatro retos fundamentales: la nueva orientación didáctica europea de educación en competencias, entre las que destaca la competencia espacial; la reducción de recursos expositivos y su sustitución por metodologías de aprendizaje más activas; la enseñanza-aprendizaje de los grandes temas sociales, políticos, económicos y culturales de la agenda mundial, actual y futura, que tienen incidencia espacial, entre otros la globalización, la sostenibilidad, el cambio climático, el desarrollo urbano, la gestión de los recursos, los movimientos migratorios, etc.; y la progresiva utilización de sistemas de información geográfica y dispositivos digitales que utilizan recursos geográficos y cartográficos, muchos de ellos de fácil acceso en la red, así como la adquisición de habilidades y métodos propios del trabajo geográfico (De Miguel et al., 2011). Dicho esto, las competencias que pueden desarrollarse gracias a esta herramienta son metodológicas, personales y competencias específicas para convivir y habitar el mundo. Entre ellas destacan el Tratamiento de la información y la competencia digital; la Competencia matemática; el Desarrollo de la autonomía e iniciativa personal; el Conocimiento y la interacción con el medio físico; y la Competencia social y ciudadana. En el caso de la ESO, además de las competencias anteriores (mucho más desarrolladas y con un nivel de exigencia mayor al alumno), deben incluirse la Competencia Expresión cultural y artística; la Competencia en Comunicación lingüística; y la Competencia para Aprender a aprender.

6. Consultar el Decreto 5/2011 de 28 de enero en el BOR. Anexo $2^{\circ}$ núm. 16, Página XXI del Boletín Oficial de La Rioja. Viernes, 4 de febrero de 2011. Consultado el 14 de junio de 2014 en, http://www.larioja.org/borO/IMG/Bor/2011/ib17.pdf. 


\subsection{Metodología. IDERioja como elemento de geo-visualización}

IDERioja es, como el resto de las infraestructuras de datos espaciales, un sistema informático integrado por un conjunto de recursos dedicados a gestionar información geográfica. Los recursos están disponibles en Internet (http://www.idee.es), cumplen unas condiciones de interoperabilidad y permiten que los usuarios, puedan emplearlos y combinarlos según sus necesidades. Aunque el Gobierno de La Rioja venía utilizando la tecnología SIG desde 1990, hasta el periodo 2003-2004 no surgió la aplicación IDERioja. Con ella, el Gobierno de La Rioja se planteó facilitar el acceso de los ciudadanos a los datos geográficos que produce y gestiona, para lo cual ha desarrollado distintas herramientas, accesibles a través de esta página? .

\section{Propuesta de actividades}

La secuenciación de las actividades propuestas se inicia con una sesión de presentación del conjunto de las prácticas y con un primer acercamiento al uso de la herramienta de trabajo. Una vez conocida la función de cada botón de la plataforma IDERioja se realiza la propuesta de actividades para el aprendizaje de aspectos relacionados con el medio urbano. Las actividades se van a realizar a escala urbana y la propuesta que se presenta se basa en estrategias de aprendizaje por descubrimiento, utilizando metodologías como la solución de problemas y agrupamientos de trabajo tanto individual y colectivo; además, se hace hincapié en la gestión y manejo de la información y exposición de resultados, para lo que se incluye una serie de ejercicios que van aumentando su nivel de dificultad y suponiendo un nivel de esfuerzo y reflexión diferente. En todas las actividades se proponen varias fases, desde la propia presentación de la actividad, el reparto del material necesario para realizarla y la forma de entrega; la realización de la misma (individual o en equipo) y la corrección por parte del profesor de los errores cometidos. Como es habitual en este tipo de ejercicios se propone una evaluación continua, integradora y contextualizada; en definitiva, ajustada a los conocidos pasos: inicial o diagnóstica, formativa y sumativa o final.

Una toma de contacto permitirá saber qué conocimientos previos del entorno inmediato tiene el alumno y cuales ha recibido a través del libro de texto y de las explicaciones del profesor. El paso siguiente es conocer con más detalle el programa con el que se va a trabajar.

\subsection{Educación Primaria}

\subsubsection{Actividades previas}

3.1.1.1. Aprendo el manejo de los botones del programa

El punto de partida es el aprendizaje del manejo de las herramientas básicas de IDERioja: identificar cada botón y conocer su uso (ver Figura 1). Los más útiles son: zoom general, ver datos de capa, desplazar el mapa, zoom de zona, ir a coordena-

7. Puede accederse también a los datos del Gobierno de La Rioja desde cualquier visualizador estándar, a través de sus servicios Open Geospatial Consortium (OGC). 
das, callejero o parcela, zoom inicial, coordenadas, medición de distancias, medición de áreas, gráficos, etiquetas, Google Street view y buscar fotografías en Panoramio. Además, junto a la pestaña Leyenda aparece la pestaña Otras capas, donde la información se encuentra organizada en carpetas que contienen datos de carácter geográfico y medioambiental muy interesantes.

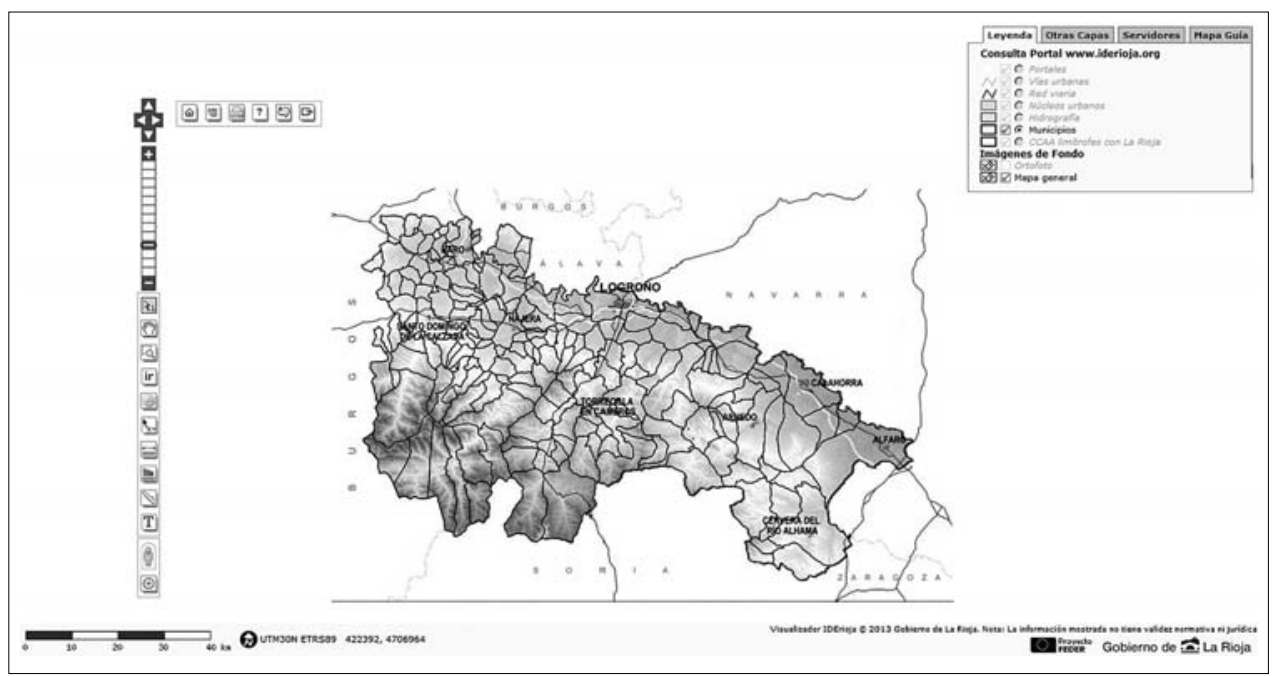

Figura 1. Imagen inicial de IDERioja

\subsubsection{Aprendo conceptos básicos de la Geografía}

Conceptos básicos: mapa, leyenda, escala, fotografía aérea, ortofoto...

De este modo y, como durante años se ha hecho con los mapas y planos en papel, el alumno identifica cada concepto y ve cómo aparece representado en las imágenes y dónde. Y, paso a paso, irá reconociendo lo que es una ortofoto y cómo se identifican y representan los diferentes hitos urbanos. Al abrir el Visualizador regional aparece un mapa de la Comunidad. Es el momento de hacer que los alumnos "jueguen" con las escalas. El objetivo es facilitar el aprendizaje de este concepto y conseguir que vean cómo se modifica el nivel de detalle cuando cambiamos de una escala pequeña a una escala grande. Podemos pedir a nuestros alumnos que busquen las comunidades autónomas limítrofes; que busquen la zona montañosa y el valle y aprendan el significado de la variable color en cartografía; el municipio en el que residen, o un lugar emblemático determinado (San Millán de la Cogolla, el pico de San Lorenzo, Calahorra, los Sotos de Alfaro, etc.). Una vez analizada la escala regional, les pedimos que se centren en cualquiera de las cabeceras comarcales ayudados del botón zoom. En este nivel el alumno debe responder a cuestiones relacionadas con las semejanzas y diferencias entre la imagen anterior y la que están viendo. Finalmente y, para pasar al tercer nivel de actividades, les pedimos que se centren en la ciudad de Logroño. 
3.1.2. Veo el plano de la ciudad: descubro y reconozco elementos básicos de la trama urbana

Conceptos a identificar: el río, el casco urbano, el casco antiguo, los ensanches, las nuevas zonas de expansión, el parcelario, las manzanas urbanas, las vías de comunicación, los edificios emblemáticos.

El mapa y el plano son dos representaciones cartográficas que se diferencian, sobre todo, por el nivel de detalle de la imagen. El objetivo de estas actividades es enseñarles a descifrar y a ubicar elementos fundamentales de los espacios urbanos (ver Figura 2).

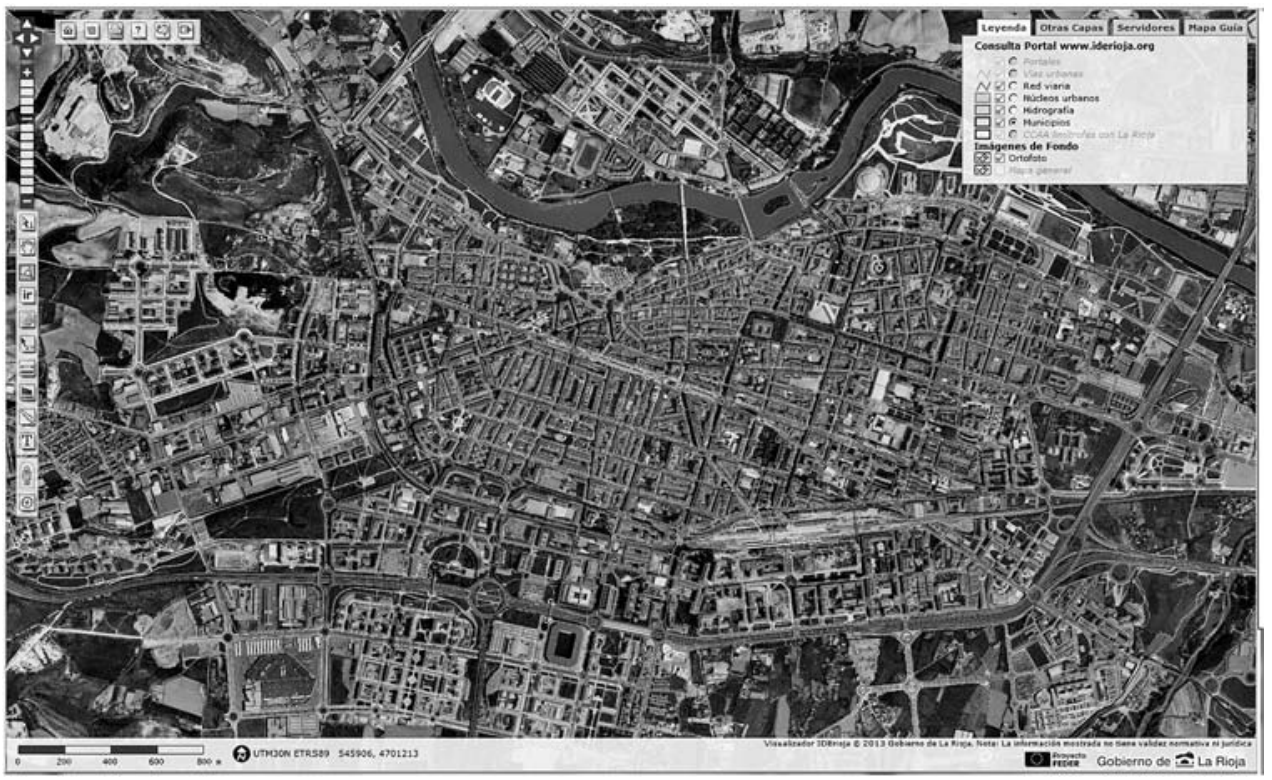

Figura 2. Vista del plano de Logroño (IDERioja)

Con el alumnado de Primaria es aconsejable empezar por aquellos que les son más fáciles de identificar o más familiares, sobre todo, haciéndoles reflexionar si estos elementos tienen un impacto directo en su entorno inmediato.

\subsubsection{Localizo/ubico, me sitúo y me oriento}

En Geografía, orientación y localización son dos conceptos fundamentales y estas herramientas de georreferenciación constituyen un apoyo fundamental para facilitar su aprendizaje. En Primaria se proponen las actividades incluidas en las tablas siguientes. 
Tabla 1. Direcciones: nos desplazamos desde varios puntos de la ciudad

\begin{tabular}{|l|l|l|l|l|l|}
\hline \multicolumn{5}{|c|}{ DIRECCIONES } \\
\hline Origen & Destino & \multicolumn{3}{|c|}{ Me desplazo } \\
\hline \multirow{4}{*}{$\begin{array}{l}\text { Macia la } \\
\text { derecha } \\
\text { domicilio }\end{array}$} & $\begin{array}{l}\text { Hacia la } \\
\text { izquierda }\end{array}$ & $\begin{array}{l}\text { Hacia } \\
\text { arriba }\end{array}$ & $\begin{array}{l}\text { Hacia } \\
\text { abajo }\end{array}$ \\
\cline { 2 - 6 } & Parque del Ebro & & & & \\
\cline { 2 - 7 } & La Redonda & & & & \\
\cline { 2 - 7 } & El Espolón & & & & \\
\hline
\end{tabular}

Tabla 2. Practicamos con los puntos cardinales

\begin{tabular}{|l|l|l|l|l|}
\hline \multicolumn{5}{|c|}{ PRACTICAMOS CON LOS PUNTOS CARDINALES } \\
\hline ¿Dónde se ubican? & Norte & Sur & Este & Oeste \\
\hline Tu colegio & & & & \\
\hline Tu domicilio & & & & \\
\hline El Ayuntamiento & & & & \\
\hline La estación del ferrocarril & & & & \\
\hline La Plaza de Toros & & & & \\
\hline
\end{tabular}

Tabla 3. Trazamos líneas sobre el plano

\begin{tabular}{|l|l|}
\hline \multicolumn{2}{|c|}{ TRAZAMOS SOBRE EL PLANO } \\
\hline \multicolumn{2}{|c|}{ Una línea norte-sur desde: } \\
\hline El Ebro hasta... & $\begin{array}{l}\text { El Espolón } \\
\text { El campo de Fútbol }\end{array}$ \\
\hline La Plaza de Toros hasta... & El Ayuntamiento \\
\hline El Ayuntamiento hasta... & La estación de ferrocarril \\
\hline & Una línea oeste-este desde: \\
\hline El Cubo del Revellín hasta... & La Redonda \\
\hline El Espolón hasta... & La Universidad de La Rioja \\
\hline Seguimos el trazado de la vía férrea \\
\hline Seguimos el trazado de la antigua muralla \\
\hline Seguimos el trazado del Parque de la Ribera
\end{tabular}


Tabla 4. Nos desplazamos por las vías de comunicación

\begin{tabular}{|l|l|}
\hline \multicolumn{2}{|c|}{ NOS DESPLAZAMOS POR LAS VíAS DE COMUNICACIÓN } \\
\hline De norte a sur siguiendo: & $\begin{array}{l}\text { Sagasta } \\
\text { Vara de Rey }\end{array}$ \\
\hline De oeste a este siguiendo: & $\begin{array}{l}\text { Avenida de la Paz } \\
\text { Gran Vía }\end{array}$ \\
\hline
\end{tabular}

\subsubsection{Localizo las actividades económicas}

En Primaria las actividades suponen una primera toma de contacto con los sectores económicos. Se localizarán los polígonos industriales y los alumnos comprueban las diferencias entre el suelo residencial y el industrial; asimismo se percatan de la diferente morfología del manzanero y, por supuesto, de la localización periférica de los polígonos (ver Tabla 5). También es interesante explicarles la ubicación de las actividades comerciales diferenciando entre el pequeño comercio y las grandes superficies o centros comerciales. En cuanto a las actividades de ocio, localizarán los parques urbanos. Con el plano delante, completarán una tabla de este tipo:

Tabla 5. Localizamos los polígonos industriales utilizando los puntos cardinales

\begin{tabular}{|l|l|l|l|l|l|l|l|l|}
\hline Localizar: & \multicolumn{7}{|c|}{$\begin{array}{c}\text { Distancia respecto al } \\
\text { centro urbano }\end{array}$} & \\
\hline Polígonos industriales & N & S & E & O & Cerca & Lejos & \\
\hline Polígono la Portalada & & & & & & & \\
\hline Polígono Cantabria I & & & & & & & \\
\hline Polígono Cantabria II & & & & & & & \\
\hline Actividades comerciales & N & S & E & O & Cerca & Lejos & \\
\hline Zonas/calles comerciales & & & & & & & & Pequeño \\
\hline Centros comerciales & & & & & & & & Tamaño \\
\hline Parques y zonas de & N & S & E & O & Cerca & Lejos & Grande & Pequen \\
\hline esparcimiento & & & & & & & & \\
\hline Parque del Ebro & & & & & & & & \\
\hline Parque de San Miguel & & & & & & & & \\
\hline Parque de la Ribera & & & & & & & & \\
\hline Parque del Carmen & & & & & & & & \\
\hline Parque González Gallarza & & & & & & & & \\
\hline
\end{tabular}

N: norte; S: sur; E: este y O: oeste 


\subsection{Educación Secundaria}

\subsubsection{Actividades previas}

En este bloque de actividades se trata, fundamentalmente, de que el alumno de Secundaria recuerde el manejo del programa y las funciones de cada botón de la plataforma IDERioja y, además, recuerde y refuerce los conocimientos adquiridos en la propuesta para tercer ciclo de Primaria.

\subsubsection{Veo el plano de la ciudad: descubro y reconozco elementos de la trama urbana}

Así como con el alumnado de Primaria las preguntas se vinculan a conceptos básicos (el río, el casco urbano, el casco antiguo, los ensanches, las nuevas zonas de expansión, el parcelario, las manzanas urbanas, las vías de comunicación, los edificios emblemáticos) y al reconocimiento en el plano de elementos de su entorno, en Secundaria se propone un nivel de reflexión y análisis mayor. Se trata de que los alumnos establezcan relaciones entre los diferentes elementos de la trama urbana y de que valoren el impacto de los mismos, no solo en su entorno inmediato, sino en el conjunto de la ciudad. Es importante que analicen y comprendan cómo ha evolucionado el plano de la ciudad a lo largo de la historia.

\subsubsection{Localizo/ubico, me sitúo y me oriento}

En Secundaria se refuerzan las ideas de localización y de orientación en el plano urbano y también en el mapa de la Comunidad con el fin de reforzar el aprendizaje del concepto de escala. En el primer caso, se insiste en la propuesta para el nivel de Primaria realizando actividades que incluyen recorridos urbanos siguiendo los puntos cardinales y a partir de hitos importantes de la ciudad (monumentos, edificios históricos, plazas, etc.). Pero, además, se proponen actividades que supongan una profundización y un esfuerzo mayor de análisis y reflexión. A modo de ejemplo: deben seguir el cauce del Ebro y señalar los puentes para comprobar la comunicación entre un lado y otro de la ciudad; seguir el trazado de la antigua muralla, localizar los ensanches, seguir el trazado del ferrocarril y comprobar las pasarelas y la parte soterrada, etc. En este nivel, la localización se plantea con las coordenadas UTM.

En el segundo caso y, con el fin de reforzar el aprendizaje del concepto pequeña/gran escala que suele resultar confuso a los alumnos de este nivel, se plantean actividades de refuerzo combinando el ámbito regional (ver Tabla 6) y la escala urbana. Se trata de que reflexionen sobre el nivel de detalle de la cartografía a diferentes escalas (por ejemplo, 1:50000, 1:5000, 1:500). 
Tabla 6. Localizamos con las coordenadas UTM lugares importantes de La Rioja

\begin{tabular}{|l|l|l|l|}
\hline Localizar & Coordenadas UTM & $\begin{array}{l}\text { Escala de trabajo } \\
\text { adecuada }\end{array}$ & $\begin{array}{l}\text { Ubicación general en el } \\
\text { mapa de La Rioja }\end{array}$ \\
\hline Pico San Lorenzo & $\begin{array}{l}\text { Ej. UTM30N ETRS89 } \\
502287,4676696\end{array}$ & & \\
\hline Embalse de Mansilla & & & \\
\hline Calahorra & & & \\
\hline $\begin{array}{l}\text { Santo Domingo de } \\
\text { la Calzada }\end{array}$ & & & \\
\hline Arnedillo & & & \\
\hline $\begin{array}{l}\text { Monasterio de } \\
\text { Valvanera }\end{array}$ & & & \\
\hline Los Sotos de Alfaro & & & \\
\hline
\end{tabular}

Una vez finalizada la localización de estos puntos relevantes a escala regional, nos centramos en Logroño y realizamos actividades de localización dentro del área urbana (ver Tabla 7 y Tabla 8). Aprovechamos también para que utilicen la herramienta de medición de superficies y les pedimos que lo anoten en la tabla (aunque el dato sea aproximado).

Tabla 7. Localizamos con las coordenadas UTM lugares importantes de la ciudad de Logroño

\begin{tabular}{|l|l|l|}
\hline Localizar & Coordenadas UTM & Superficie (aproximada) \\
\hline El Ayuntamiento & $\begin{array}{l}\text { Ej. UTM30N ETRS89 } \\
546079,4701727\end{array}$ & \\
\hline Palacio de los Deportes & & \\
\hline Embalse de la Grajera & & \\
\hline Monte Cantabria & & \\
\hline
\end{tabular}

Tabla 8. Identificamos nuestra ubicación en la ciudad con las coordenadas UTM

\begin{tabular}{|l|l|}
\hline Coordenadas UTM & ¿Dónde estás? \\
\hline UTM30N ETRS89 54495, 4700240 & \\
\hline UTM30N ETRS89 545287, 4702039 & \\
\hline UTM30N ETRS89 543654, 4700798 & \\
\hline
\end{tabular}

Después de realizar estas tareas es necesario repasar las ideas generales sobre el concepto de escala y exponer los problemas que el alumnado ha tenido para localizar en el plano hitos importantes y para ubicarse. 


\subsubsection{Localizo las actividades económicas}

El alumnado de Secundaria debe no solo identificar, sino diferenciar y ubicar correctamente los diversos usos de suelo (suelo industrial, residencial, dotacional), la localización de los servicios y del comercio y conocer cuestiones básicas de la morfología urbana. Por eso, además de localizar, como se propone para Primaria, es conveniente que marquen sobre el plano la superficie de los polígonos industriales utilizando la herramienta de medición de superficies (ver Tabla 9).

Tabla 9. Localizamos los polígonos industriales utilizando las coordenadas UTM

\begin{tabular}{|l|l|l|l|l|}
\hline Localizar & $\begin{array}{l}\text { Coordenadas } \\
\text { UTM }\end{array}$ & $\begin{array}{l}\text { Superficie } \\
\text { (aproximada) }\end{array}$ & $\begin{array}{l}\text { Tipo de empresa } \\
\text { dominante }\end{array}$ & $\begin{array}{l}\text { Fecha de } \\
\text { instalación }\end{array}$ \\
\hline La Portalada & & & & \\
\hline Cantabria I & & & & \\
\hline Cantabria II & & & & \\
\hline Polígono industrial El Cortijo & & & & \\
\hline Polígono de San Lázaro & & & & \\
\hline
\end{tabular}

De la misma forma que se trabaja con el tejido industrial, deben analizar el resto de tipos de suelo y ofrecer una explicación de dónde se ubican las actividades comerciales, los servicios más importantes (hospitales, centros educativos y administración autonómica), así como de su nivel de centralidad (proximidad al centro) y accesibilidad desde las vías de comunicación. También es preciso que marquen sobre el plano estas zonas (ver Tabla 10).

Tabla 10. Localizamos las actividades comerciales y de servicios

\begin{tabular}{|c|c|c|c|c|c|c|c|}
\hline \multirow[t]{2}{*}{ Localizar } & \multirow[t]{2}{*}{$\begin{array}{l}\text { Coordenadas } \\
\text { UTM }\end{array}$} & \multicolumn{3}{|c|}{ Centralidad } & \multicolumn{3}{|c|}{$\begin{array}{l}\text { Accesibilidad desde } \\
\text { las vías de } \\
\text { comunicación }\end{array}$} \\
\hline & & A & $\mathbf{M}$ & B & A & $M$ & B \\
\hline \multicolumn{8}{|l|}{ Actividades comerciales } \\
\hline \multicolumn{8}{|l|}{ Zonas/calles comerciales } \\
\hline \multicolumn{8}{|l|}{ Centros comerciales } \\
\hline \multicolumn{8}{|l|}{ Servicios importantes } \\
\hline \multicolumn{8}{|l|}{ Hospitales } \\
\hline \multicolumn{8}{|l|}{$\begin{array}{l}\text { Sede del Gobierno } \\
\text { autonómico }\end{array}$} \\
\hline $\begin{array}{l}\text { Centros educativos de } \\
\text { Primaria y Secundaria }\end{array}$ & & & & & & & \\
\hline
\end{tabular}

A: Alta; M: Media y B: Baja 
Pero, una vez realizadas estas tareas prácticas, es necesaria una reflexión que responda a preguntas tales como: ¿dónde se ubican las actividades económicas?, ¿por qué?, ¿de qué tipo son?, ¿generan algún impacto negativo?, ¿cuál?, ¿dónde? En esta fase resultaría interesante que el alumno realizara actividades complementarias de búsqueda de información sobre las características de las empresas ubicadas en la periferia urbana (se trata de grandes empresas o de empresas familiares, son manufactureras, conserveras, etc.), datos de producción históricos si los hubiera, principales relaciones comerciales, etc.

Además, ha de responder a cuestiones relacionadas con la evolución del plano y explicar hacia dónde ha crecido el casco urbano y por qué. La unidad más representativa es el casco histórico, pero también deben señalar otras zonas de expansión urbana contiguas al mismo, o de localización periférica. Se trata de que definan e identifiquen el tipo de plano (lineal, ortogonal, irregular, concéntrico) y de que reflexionen sobre el tipo de edificación característico de cada etapa de expansión (manzana abierta, manzana cerrada, bloque, unifamiliar), así como sobre el uso de suelo predominante en cada sector (residencial, comercial, dotacional). Deben marcar sobre el plano estas zonas (ver Tabla 11).

Tabla 11. Localizamos el casco histórico y otras zonas de la ciudad

\begin{tabular}{|c|c|c|c|c|c|c|c|c|c|c|}
\hline \multirow[t]{2}{*}{ Localizar } & \multirow[t]{2}{*}{$\begin{array}{l}\text { Coordenadas } \\
\text { UTM }\end{array}$} & \multirow[t]{2}{*}{$\begin{array}{l}\text { Tipo de } \\
\text { edificación }\end{array}$} & \multirow[t]{2}{*}{$\begin{array}{l}\text { Uso del suelo } \\
\text { dominante }\end{array}$} & \multirow[b]{2}{*}{ Fecha } & \multicolumn{3}{|c|}{ Centralidad } & \multicolumn{3}{|c|}{ Accesibilidad } \\
\hline & & & & & A & $M$ & B & $\mathrm{A}$ & $M$ & B \\
\hline \multicolumn{11}{|l|}{ Casco histórico } \\
\hline \multicolumn{11}{|l|}{$\begin{array}{l}\text { Centro de } \\
\text { negocios }\end{array}$} \\
\hline $\begin{array}{l}\text { Zonas de } \\
\text { expansión }\end{array}$ & & & & Fecha & A & M & B & A & M & B \\
\hline \multicolumn{11}{|l|}{ Cascajos } \\
\hline \multicolumn{11}{|l|}{ El Cubo } \\
\hline \multicolumn{11}{|l|}{ Los Lirios } \\
\hline \multicolumn{11}{|l|}{$\begin{array}{l}\text { La Cava- } \\
\text { Fardachón }\end{array}$} \\
\hline \multicolumn{11}{|l|}{ El Arco } \\
\hline \multicolumn{11}{|l|}{ San Adrián } \\
\hline \multicolumn{11}{|l|}{ Avda. de Madrid } \\
\hline El Campillo & & & & & & & & & & \\
\hline
\end{tabular}

A: Alta; M: Media y B: Baja

Es también conveniente que estos alumnos analicen las relaciones entre el crecimiento de la ciudad, el reparto de los usos del suelo y los problemas de accesibilidad y movilidad que se han generado en las diferentes áreas urbanas.

Finalmente, el alumno debe conocer su área de desplazamiento cotidiana, reflexionando sobre los motivos (trabajo, estudios, cuestiones administrativas, de salud, ocio y cultura) y las características y/o patrón de los desplazamientos que realiza en 
función de si son obligatorios o puntuales. Se apuesta por una profundización en el conocimiento que los alumnos tienen de su "barrio" o del entorno de su lugar de residencia: es antiguo o moderno, las casas son unifamiliares o son bloques de viviendas, los servicios son adecuados o no comparados con los de sus compañeros, está bien comunicado o existen problemas de accesibilidad, etc.

El nivel de madurez exigido en este bloque es, como se puede comprobar, mayor dado que las actividades propuestas no son sólo de localización y de medición de distancias y áreas recorridas; de hecho, suponen un conocimiento más profundo del entorno del estudiante (fortalezas y debilidades), así como del conjunto de la ciudad y de su funcionamiento como sistema.

\subsubsection{Actividades complementarias}

Se trata de presentar un análisis completo del paisaje urbano. Ya se han descrito y localizado los principales elementos de la ciudad y ahora tienen que ser capaces de mostrar sus habilidades para ofrecer una visión global del espacio urbano. La actividad combinará el juego de simulación y la resolución de problemas, dos estrategias atractivas, que despiertan el interés del alumnado y que ayudan al desarrollo del conocimiento social. La propuesta de actividades se centrará en i) cuestiones de población: debate entre alumnos que desempeñan el papel de representantes de la población local y de la población inmigrante, miembro de una ONG, un concejal de familia y política social; ii) otras cuestiones sociales: la situación del casco antiguo (un residente en el casco antiguo, un residente en un barrio periférico, un representante de las asociaciones de vecinos, un concejal de distrito), los atascos en horas punta (un taxista, un repartidor, un peatón, un concejal de movilidad, un concejal de medio ambiente), la falta de servicios en algunos barrios (un concejal de distrito, representantes de las asociaciones de vecinos, un comerciante, un concejal de comercio, cultura y turismo); iii) cuestiones medioambientales (un representante de las asociaciones de vecinos, un concejal de medio ambiente, un vecino afectado por vertidos incontrolados, un vecino afectado por contaminación acústica).

Una vez expuestos y analizados los problemas, se plantea una discusión sobre soluciones posibles. El papel del profesor como guía, moderador y responsable de la calidad de la actividad es especialmente importante en esta fase.

\subsection{Criterios de evaluación}

Tanto en Primaria como en Secundaria se entregarán todos los ejercicios debidamente referenciados en un cuadernillo o portafolio. Se tendrá en cuenta tanto la presentación como el grado de implicación del alumnado en el desarrollo de los mismos. Desde el punto de vista de los contenidos, en Primaria se valorará la habilidad del alumno para manejar la IDE; la habilidad para localizar elementos básicos del espacio urbano; la habilidad para interpretar la leyenda y otros símbolos; la habilidad para describir el entorno en el que se desarrolla su vida cotidiana, así como su capacidad para interpretar algunos efectos de la intervención del hombre. En Secundaria el manejo de la IDE ha de ser más completo. En este caso, se han de valorar también cuestiones de localización pero a un nivel de detalle más profundo y demostrando, sobre todo, su capacidad de análisis y de síntesis. Además, ha de 
tenerse en cuenta la capacidad para reconocer los espacios residenciales, industriales, comerciales y su ubicación; deben saber cómo obtener y analizar información socioeconómica básica para explicar los desequilibrios dentro de las áreas urbanas. En esa labor de síntesis mencionada deben saber aportar explicaciones a los hechos sociales. En todos los casos, es importante una cuestión más: la realización de una autoevaluación del trabajo realizado, desde el planteamiento hasta la obtención de resultados.

\section{Conclusiones}

Las herramientas geo-espaciales, entre las que se encuentran las IDES, constituyen un instrumento muy versátil e interesante para explicar cuestiones básicas de Geografía. Estas infraestructuras de datos espaciales se utilizan en todo el mundo para estudios medioambientales fundamentalmente. Sin embargo, su uso se ha extendido a las administraciones locales y autonómicas. En este sentido, hay que destacar el ejemplo de la Unión Europea, que ha realizado un gran esfuerzo para conseguir armonizar la información geográfica y que ésta pueda ser compartida por diferentes países. A escala nacional, España se ha subido al carro de esta iniciativa y todas las comunidades autónomas disponen de una IDE. Los datos son utilizados también por las administraciones locales y ofrecen al ciudadano la posibilidad de acceder de manera rápida y sencilla a las bases de datos disponibles. Pero además, las IDEs tienen posibilidades de aplicación didáctica y constituyen una herramienta interesante para que los alumnos desarrollen sus habilidades cartográficas, potencien la competencia digital y mejoren en las cuestiones de interpretación de la información geográfica. Para un alumnado familiarizado con Internet, los videojuegos y las tecnologías más modernas esta herramienta, convenientemente combinada con las actividades que tradicionalmente se desarrollan en las aulas, contribuye a agilizar y facilitar el aprendizaje de conceptos importantes en el ámbito geográfico como son la escala, la localización, la orientación, y los usos del suelo entre otros. Son, por lo tanto, un complemento fundamental para facilitar el aprendizaje de los contenidos teóricos de una asignatura que, de entrada, no suele resultar atractiva. Los profesores de Primaria y Secundaria deben promover el aprendizaje activo aprovechando estos recursos informáticos básicos, adaptándolos al nivel del alumnado y buscando la manera de atraer al discente para que sea él mismo quién plantee nuevas preguntas, proponga soluciones y desarrolle su capacidad crítica a la hora de evaluar cualquier cuestión de carácter social. En este caso, en temas urbanos.

\section{REFERENCIAS BIBLIOGRÁFICAS}

ARRANZ, A.; PUEYO, A. y ZÚÑIGA, M. (2012). Las herramientas de geovisualización para el aprendizaje de la geografía. En F. R. Durán, R. López, M. C. Saavedra, J. A. Sánchez y M. Villarino (Coords.), Innovación metodológica y docente en historia, arte y geografía (Recurso electrónico). Actas del Congreso Internacional de Santiago de Compostela. Santiago de Compostela: Universidade de Santiago de Compostela, 205-218. 
BERNARD, L.; KANELLOPOULOS, I.; ANNONI, A. y SMITS, P. (2005). The European geoportal-one step towards the establishment of a European Spatial Data Infraestructure. Computers, Environment and Urban Systems, 29, 15-31.

CAPDEVILA I SUBIRANA, J. (2004). Infraestructura de Datos Espaciales (IDE). Definición y desarrollo actual en España. Scripta Nova, Revista electrónica de Geografía y Ciencias Sociales, Vol. VIII, $n^{\circ} 170$ (61).

CAPEL, H. (2010). Geografía en red a comienzos del tercer milenio: para una ciencia solidaria y en colaboración. Scripta Nova, Revista electrónica de Geografía y Ciencias Sociales, Vol. XIV, no 313, 69 pp.

CARCELLER, T.; MIGUEL, R.; LOSADA, J. A.; VIDAL, M.; GAYÁN, D. y ZARAGOZA-SORIA, F. J. (2009). IDE-Ebro: La infraestructura de Datos Espaciales de la Confederación Hidrográfica del Ebro. Mapping, 135, 79-86.

DE ALBA, N.; GARCÍA, F. y SANTISTEBAN, A. (Eds.) (2012). Educar para la participación ciudadana en la enseñanza de las Ciencias Sociales. Sevilla: Asociación Universitaria de Profesorado de Didáctica de las Ciencias Sociales.

DE MIGUEL, R.; DE LÁZARO, M. L. y MARRÓN, M. J. (Eds.) (2012). La educación geográfica digital (CD-ROM). Zaragoza: Grupo de Didáctica de la Geografía de la AGE.

DELGADO, J. J.; DE LÁZARO, M. L. y MARRÓN, M. J. (Coords.) (2011). Aportaciones de la Geografía a lo largo de la vida. Congreso Ibérico de Didáctica de la Geografía. Málaga: Universidad de Málaga: Asociación de Geográfos Españoles.

DELVAL, J. (2012). Ciudadanía y escuela. El aprendizaje de la participación. En N. de Alba, F. García y A. Santisteban (Eds.), Educar para la participación ciudadana en la enseñanza de las Ciencias Sociales. Vol. I. Sevilla: Asociación Universitaria de Profesorado de Didáctica de las Ciencias Sociales, 37-46.

DÍEZ, M. C.; GÁMEZ, M. D.; CRUZ, M. A. y RUEDA, C. (2004). Las TICs y su aplicación en el área de Ciencias sociales. En M. I. Vera y D. Pérez (Coords.), Formación de la ciudadanía: las TICs y los nuevos problemas. Simposio Internacional de Didáctica de las Ciencias Sociales. Alicante: Asociación Universitaria de Profesores de Didáctica de las Ciencias Sociales.

DURÁN, F. R.; LÓPEZ, R.; SAAVEDRA, M. C.; SÁNCHEZ, J. A. y VILLARINO, M. (Coords.) (2012). Innovación metodológica y docente en historia, arte y geografía (recurso electrónico). Actas del Congreso Internacional de Santiago de Compostela. Santiago de Compostela: Universidade de Santiago de Compostela.

GARCÍA, T. y PASCUAL, N. (2012). Posibilidades de la plataforma IDERioja para el análisis de la ciudad de Logroño. Un proyecto para tercer ciclo de Educación Primaria. En R. de Miguel, M. L. de Lázaro y M. J. Marrón (Eds.), La educación geográfica digital (CD-ROM). Zaragoza: Grupo de Didáctica de la Geografía de la AGE, 167-178.

GENOVART, M. C. (2011). La enseñanza de la Geografía mediante el uso de las TIC. En J. J. Delgado, M. L. de Lázaro, y M. J. Marrón (Coords.), Aportaciones de la Geografía a lo largo de la vida. Congreso Ibérico de Didáctica de la Geografía. Málaga: Universidad de Málaga: Asociación de Geográfos Españoles, 292-301.

GOBIERNO DE LA RIOJA. Infraestructura de Datos Espaciales [en línea]. Disponible en: http://www.iderioja.larioja.org/. 
GÓMEZ, M. I. (2010). Análisis del paisaje físico y humano de la provincia de Alicante: Google Earth como herramienta docente en las clases de Geografía. Geographos. Revista digital para estudiantes de geografía y Ciencias Sociales, Vol. 1 (1). Disponible en: http://web.ua.es/es/revista-geographos-giecryal/volumen1-2010/revista-geographos-grupo-interdisciplinario-de-estudios-criticos-y-deamerica-latina-giecryal.html.

GÓMEZ, M. L. (2011). Los cambios en el medio urbano como instrumento didáctico para trabajar la percepción espacial. En J. J. Delgado, M. L. de Lázaro y M. J. Marrón (Coords.), Aportaciones de la Geografía a lo largo de la vida. Congreso Ibérico de Didáctica de la Geografía. Málaga: Universidad de Málaga: Asociación de Geográfos Españoles, 488-502.

LAND, N. (2004). El papel de las Agencias Cartográficas Nacionales en la construcción de las Infraestructuras de Datos Espaciales de Europa. Topografía y Cartografía. Revista del Ilustre Colegio Oficial de Ingenieros Técnicos en Topografía, Vol. XXI (121), 30-34.

MAS, S. (2002). Propuesta para el establecimiento de la Infraestructura de Datos Espaciales de España (IDEE). Mapping, 77, 6-18.

MAS, S. y VALLEJO, R. (2003). El proyecto de Directiva europea INSPIRE. Mapping, $86,45-49$.

MEJÍA, D. (2008). Sistemas de Información Geográfica, Infraestructura de Datos espaciales y educación. Mapping, 125, 42-49.

MINISTERIO DE FOMENTO. Consejo Superior Geográfico. Infraestructura de datos espaciales de España [en línea]. Disponible en: http://www.idee.es.

MORAGA, J. (2011). Aprendizaje invisible: hacia una nueva didáctica de la Geografía. En J. J. Delgado, M. L. de Lázaro y M. J. Marrón (Coords.), Aportaciones de la Geografía a lo largo de la vida. Congreso Ibérico de Didáctica de la Geografía. Málaga: Universidad de Málaga : Asociación de Geográfos Españoles, 130-145.

NOGUERAS-ISO, J.; ZARAGOZA-SORIA, F. J.; BÉJAR, R.; ÁLVAREZ, P. J. y MUROMEDRANO, P. R. (2005). OGC Catalog Services: a key element for the development of Spatial data Infraestructures. Computers \& Geosciences, 31, 199-209.

PÉREZ, D. y VERA, M. I. (2004). El profesorado en formación de Ciencias Sociales y las TICs: situación y perspectivas. En M. I. Vera y D. Pérez (Coords.), Formación de la ciudadanía: las TICs y los nuevos problemas. Disponible en: http://dialnet. unirioja.es/descarga/articulo/1448326.pdf.

RODRÍGUEZ, A. F.; ABAD, P.; ALONSO, J. A.; ZAMORANO, C.; DE CARVALHO, C.; CABALLERO, C. y CEBALLOS-ZÚÑIGA, F. (2008). La IDEE y el desarrollo sostenible. Mapping, 125, 12-19.

ROMERO, O. L. La ciudad: un espacio local contextualizado (estudio de caso: estrategias pedagógicas para el aprendizaje y enseñanza del espacio geográfico urbano). Disponible en: http://observatoriogeograficoamericalatina.org.mx/egal11/ Geografiasocioeconomica/Geografiaurbana/34.pdf.

SORIANO, M. C. (2004). Las TICs en el profesorado en ejercicio de Ciencias Sociales: análisis de sus conocimientos y necesidades de formación. En M. I. Vera y D. Pérez (Coords.), Formación de la ciudadanía: las TICs y los nuevos problemas. Disponible en: http://dialnet.unirioja.es/descarga/articulo/1448512.pdf. 
VALVUENA, M. y VALVERDE, J. A. (2004). El uso de las TICs como aplicación didáctica para la formación inicial del maestro. En M. I. Vera y D. Pérez (Coords.), Formación de la ciudadanía: las TICs y los nuevos problemas. Disponible en: http:// dialnet.unirioja.es/servlet/libro?codigo $=8688$.

VELARDE, J. et al. (2012). Nuevos aires en la Geografía española del siglo XXI (CDROM). Contribución española al $32^{\circ}$ Congreso de la Unión Geográfica Internacional. Madrid: Comité Español de la UGl.

VERA, M. I. y PÉREZ, D. (Coords.) (2004). Formación de la ciudadanía: las TICs y los nuevos problemas. Simposio Internacional de Didáctica de las Ciencias Sociales. Alicante: Asociación Universitaria de Profesores de Didáctica de las Ciencias Sociales.

ZAPATA, T. (2011). El proyecto ESDIN: el paso de la teoría a la práctica; de la Directiva INSPIRE a la implementación de la infraestructura de Datos Espaciales Europea. CT/Catastro, 72, 61-72. 